\title{
Pipeline Embolization Device for small paraophthalmic artery aneurysms with an emphasis on the anatomical relationship of ophthalmic artery origin and aneurysm
}

\author{
Christoph J. Griessenauer, MD, ${ }^{1}$ Christopher S. Ogilvy, MD, ${ }^{1}$ Paul M. Foreman, MD, ${ }^{2}$ \\ Michelle H. Chua, BS, ${ }^{3}$ Mark R. Harrigan, MD, ${ }^{2}$ Christopher J. Stapleton, MD, ${ }^{4}$ Aman B. Patel, MD, ${ }^{4}$ \\ Lucy He, MD, ${ }^{5}$ Matthew R. Fusco, MD, ${ }^{5}$ Mocco, MD, MS, ${ }^{6}$ Peter A. Winkler, MD, ${ }^{7}$ \\ Apar S. Patel, MD, MPH, ${ }^{1}$ and Ajith J. Thomas, MD${ }^{1}$ \\ ${ }^{1}$ Neurosurgical Service, Beth Israel Deaconess Medical Center, Harvard Medical School; ${ }^{3}$ Harvard Medical School; ${ }^{4}$ Department \\ of Neurosurgery, Massachusetts General Hospital, Harvard Medical School, Boston, Massachusetts; ${ }^{2}$ Department of \\ Neurosurgery, University of Alabama at Birmingham, Alabama; ${ }^{5}$ Department of Neurosurgery, Vanderbilt University, Nashville, \\ Tennessee; ${ }^{6}$ Department of Neurosurgery, Mount Sinai, New York, New York; and ${ }^{7}$ Department of Neurosurgery, Paracelsus \\ Medical University, Salzburg, Austria
}

OBJECTIVE Contemporary treatment for paraophthalmic artery aneurysms includes flow diversion utilizing the Pipeline Embolization Device (PED). Little is known, however, about the potential implications of the anatomical relationship of the ophthalmic artery $(\mathrm{OA})$ origin and aneurysm, especially in smaller aneurysms.

METHODS Four major academic institutions in the United States provided data on small paraophthalmic aneurysms $(\leq 7 \mathrm{~mm})$ that were treated with PED between 2009 and 2015. The anatomical relationship of OA origin and aneurysm, radiographic outcomes of aneurysm occlusion, and patency of the OA were assessed using digital subtraction angiography. OA origin was classified as follows: Type 1, OA separate from the aneurysm; Type 2, OA from the aneurysm neck; and Type 3, OA from the aneurysm dome. Clinical outcome was assessed using the modified Rankin Scale, and visual deficits were categorized as transient or permanent.

RESULTS The cumulative number of small paraophthalmic aneurysms treated with PED between 2009 and 2015 at the 4 participating institutions was 69 in 52 patients ( $54.1 \pm 13.7$ years of age) with a male-to-female ratio of 1:12. The distribution of OA origin was $72.5 \%$ for Type 1, 17.4\% for Type 2, and $10.1 \%$ for Type 3. Radiographic outcome at the last follow-up (median 11.5 months) was available for 54 aneurysms (78.3\%) with complete, near-complete, and incomplete occlusion rates of $81.5 \%, 5.6 \%$, and $12.9 \%$, respectively. Two aneurysms $(3 \%)$ resulted in transient visual deficits, and no patient experienced a permanent visual deficit. At the last follow-up, the OA was patent in $96.8 \%$ of treated aneurysms. Type 3 OA origin was associated with a lower rate of complete aneurysm occlusion $(p=0.0297)$, demonstrating a trend toward visual deficits $(p=0.0797)$ and a lower rate of OA patency $(p=0.0783)$.

CONCLUSIONS Pipeline embolization treatment of small paraophthalmic aneurysms is safe and effective. An aneurysm where the $\mathrm{OA}$ arises from the aneurysm dome may be associated with lower rates of aneurysm occlusion, $\mathrm{OA}$ patency, and higher rates of transient visual deficits.

http://thejns.org/doi/abs/10.3171/2015.12.JNS152499

KEY WORDS Pipeline Embolization Device; ophthalmic artery; aneurysm; occlusion; patency; vision; outcome; vascular disorders

$\mathrm{T}$ HE flow-diverting Pipeline Embolization Device (PED) (Covidien-ev3) results in the occlusion of intracranial aneurysms by disrupting blood flow into the aneurysm, leading to thrombosis followed by luminal reconstruction of the parent artery. Of concern is the preservation of the perforating arteries and side branches covered by the PED. Multiple factors govern aneurysm occlusion and branch preservation. As opposed to the aneurysm itself, the pressure gradient between the parent vessel and its branches creates an aspiration effect that al-

ABBREVIATIONS ICA = internal carotid artery; $m R S=$ modified Rankin Scale; $O A=$ ophthalmic artery; PED = Pipeline Embolization Device; SAH = subarachnoid hemorrhage; UCAS = Unruptured Cerebral Aneurysm Study. SUBMITTED October 28, 2015. ACCEPTED December 4, 2015.

INCLUDE WHEN CITING Published online March 4, 2016; DOI: 10.3171/2015.12.JNS152499. 

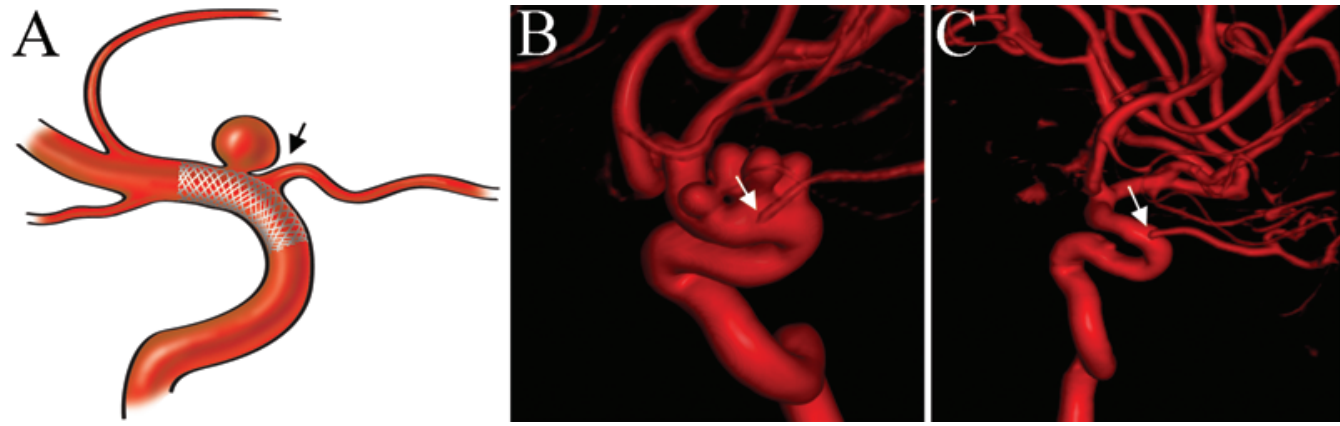

FIG. 1. Type 1: OA separate from aneurysm. A: An anatomical illustration of the $O A$ arising separate from the aneurysm. B and C: 3D angiograms of a paraophthalmic aneurysm before and after treatment with PED with preserved patency of the OA.

lows for the continuation of flow after the placement of the PED. This mechanism, however, appears to be dependent on the degree of collateralization..$^{5,10}$ In situations of robust collateral circulation, the aforementioned pressure gradient may be abolished and lead to branch occlusion; this is occasionally seen when PED is deployed across the origin of the ophthalmic artery $(\mathrm{OA})$. The notion that $\mathrm{OA}$ occlusion will only occur when sufficient collaterals from the external carotid artery are present is supported by the observation that no clinically overt visual consequences develop in the majority of these cases. ${ }^{15}$

Another important consideration in the endovascular treatment of paraophthalmic aneurysms is the anatomical relationship between OA origin and the aneurysm. Particularly in small paraophthalmic aneurysms, the OA can originate from the aneurysm dome. Coil embolization is a less attractive option for aneurysms in which the OA arises from the dome because of the possibility of the immediate occlusion of the OA origin and the potential for retinal ischemia.

We present a retrospective multicenter series of small paraophthalmic artery aneurysms that were treated with PED with an emphasis on the radiographic and clinical outcomes as they pertain to the anatomical relationship between the OA origin and the aneurysm.

\section{Methods}

Four major academic institutions in the US contributed data on consecutive patients with small $(\leq 7 \mathrm{~mm})^{20}$ aneurysms of the paraophthalmic segment of the internal carotid artery (ICA) that were treated with PED between 2009 and 2015. Paraophthalmic segment aneurysms include transitional, ophthalmic, and hypophyseal ICA aneurysms, as well as aneurysms of the dorsal wall of the ICA and carotid cave. ${ }^{3,6,11}$ Institutional review board approval was obtained at all 4 participating centers. Ruptured, dissecting, and fusiform aneurysms were included. Using a prospectively maintained database, the participating institutions provided the following information: demographics (sex, age, active smoking status, hypertension status, family history), aneurysm features (size, side and location, rupture status, history of prior treatment, morphology, intraluminal thrombus), and treatment characteristics (number of PEDs, antiplatelet regimen, use of platelet function assay, complications). The anatomical relationship between the OA origin and aneurysm, radiographic outcomes at 6 months and thereafter, and patency of the OA were assessed using digital subtraction angiography. Follow-up imaging was performed at the discretion of the individual institution. OA origin was classified as follows: Type 1, OA separate from the aneurysm (Fig. 1); Type 2, OA from the aneurysm neck (Fig. 2); and Type 3, OA from the aneurysm dome (Fig. 3). Aneurysm occlusion was categorized as complete occlusion (100\%), near complete $(\geq 90 \%)$, or incomplete $(<90 \%)$, as previously described. ${ }^{2}$ OA patency was assessed at the last radiographic follow-up. Clinical outcome was recorded using the modified Rankin Scale (mRS) at the last follow-up. Visual deficits were categorized as transient or permanent. Patients were specifically asked about visual deficits immediately post-PED placement and at the follow-up visits. Routine visual examinations were also performed. Any patient who reported visual compromise or deficit on ex-

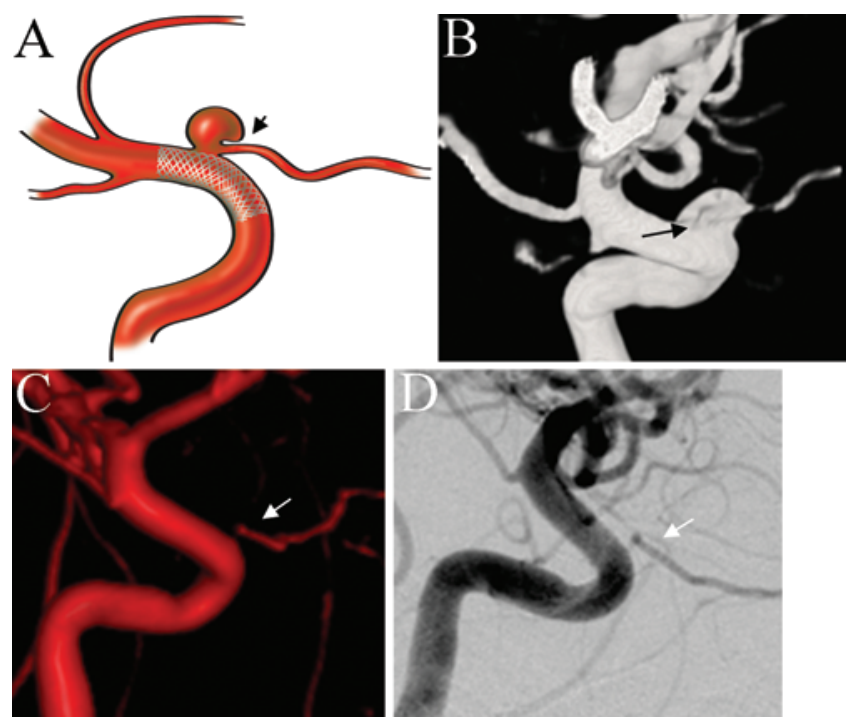

FIG. 2. Type 2: OA from aneurysm neck. A: An anatomical illustration of the $\mathrm{OA}$ arising from the aneurysm neck. B: $\mathrm{A} 3 \mathrm{D}$ angiographic view of a paraophthalmic aneurysm before treatment with PED. Six-month followup 3D (C) and lateral (D) angiographic views show aneurysm occlusion with patency of the $O A$. 

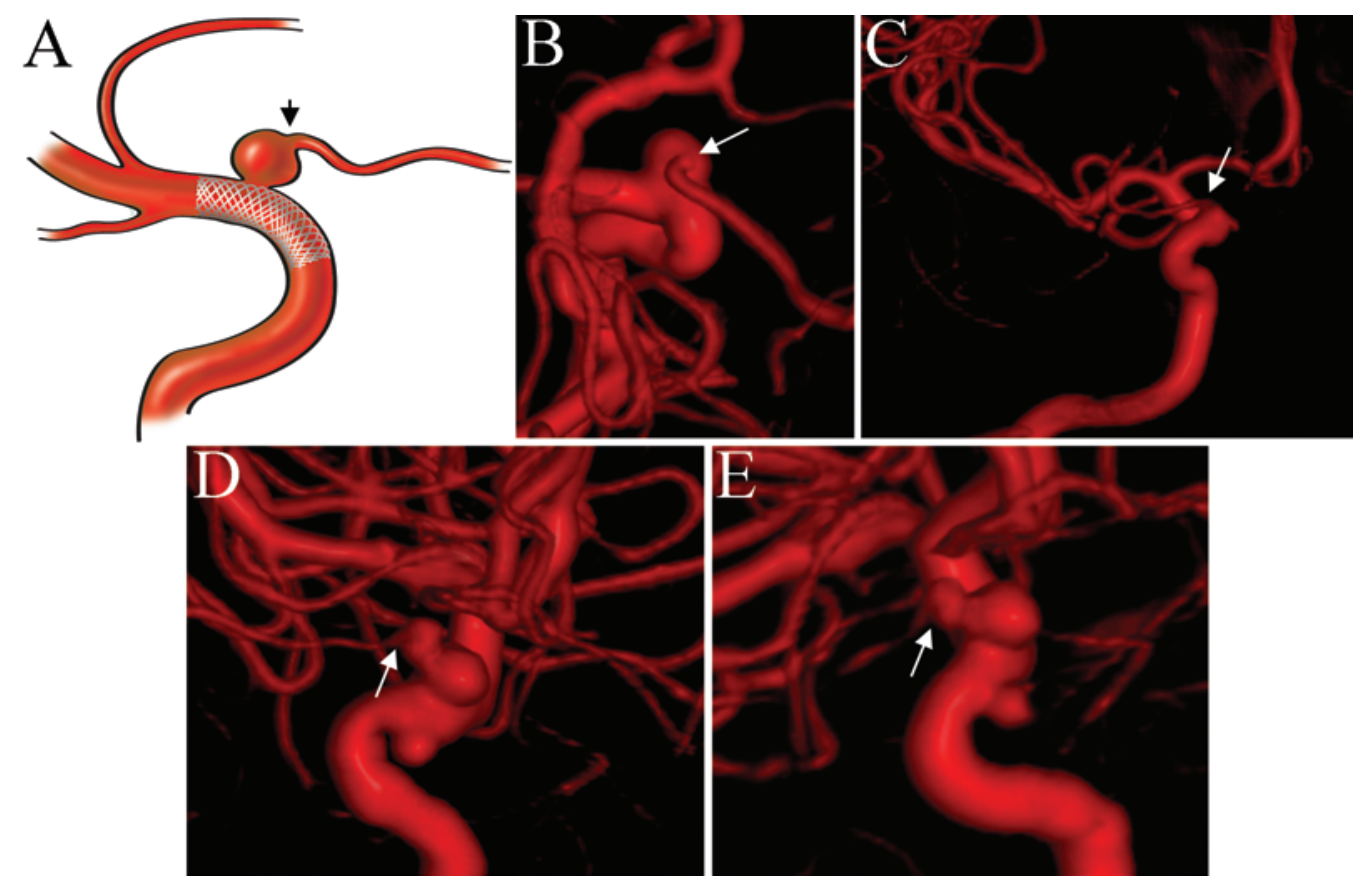

FIG. 3. Type 3: OA from aneurysm dome. A: Anatomical illustration of the $O A$ arising from the aneurysm dome. B and C: 3D views of a paraophthalmic aneurysm before and after successful treatment with PED, respectively. The OA remained patent. D: 3D views of a paraophthalmic aneurysm before PED treatment. E: On the 6-month follow-up angiogram, the aneurysm has not occluded and the OA remains patent.

amination was referred for a formal neuroophthalmological evaluation.

\section{Implantation Procedure}

Patients undergoing elective treatment with a PED received 81 or $325 \mathrm{mg}$ aspirin daily and $75 \mathrm{mg}$ clopidogrel daily for 10 to 14 days prior to intervention. Platelet function assays were performed at the discretion of the individual institution. Clopidogrel nonresponders were identified based on the protocols established at the individual institutions. Antiplatelet therapy for clopidogrel nonresponders was determined at the discretion of the individual institution. Patients undergoing treatment of a ruptured aneurysm received a loading dose of $650 \mathrm{mg}$ aspirin and 600 mg clopidogrel prior to intervention. Patients were anticoagulated with heparin throughout the procedure. The PEDs were delivered through a Marksman Micro Catheter (Covidien-ev3). The guide catheter used was at the discretion of the individual institutions. The deployment and apposition of the PED to the ICA wall was documented by fluoroscopy. Dual antiplatelet therapy was continued for at least 3 to 6 months after the procedure, and aspirin was administered indefinitely thereafter.

\section{Statistical Analysis}

Statistical analysis was performed using $\mathrm{R}$ (version 3.1.1) (http://www.r-project.org). In the univariate analysis, the variables were compared between groups by 1-way ANOVA for numerical variables and the Fisher exact test for categorical variables. Statistical significance was defined as $\mathrm{p}<0.05$.

\section{Results}

The cumulative number of small paraophthalmic aneurysms treated with PED between 2009 and 2015 at the 4 participating institutions was 69 in 52 patients (mean age $54.1 \pm 13.7$ years; male:female ratio 1:12) (Table 1). Multiple paraophthalmic aneurysms were treated in $21.2 \%$ of patients. Smoking, hypertension, and family history of aneurysm were reported in $32.7 \%, 42.3 \%$, and $15.4 \%$ of patients, respectively. The mean maximum dimension of the aneurysms was $4.9 \pm 1.6 \mathrm{~mm}$. A total of $8.7 \%$ of the aneurysms were ruptured. Saccular morphology and intraluminal thrombus were present in $94.2 \%$ and $4.3 \%$ of patients, respectively. A single PED was used in $95.7 \%$ of aneurysms. A combination of 81 or 325 aspirin mg daily and $75 \mathrm{mg}$ clopidogrel daily was the most common antiplatelet regimen (75.4\%), followed by 81 or $325 \mathrm{mg}$ aspirin daily and $90 \mathrm{mg}$ ticagrelor twice daily (14.5\%). Platelet aggregation studies identified $21.7 \%$ of patients as clopidogrel nonresponders. Radiographic outcome at the last followup (median 11.5 months) was available for 54 aneurysms (78.3\%) with a complete, near-complete, and incomplete occlusion rates of $81.5 \%, 5.6 \%$, and $12.9 \%$, respectively. The distribution of OA origin was $72.5 \%$ for Type 1 , $17.4 \%$ for Type 2, and $10.1 \%$ for Type 3 . At the last follow-up, the OA was patent in $96.8 \%$ of treated aneurysms. Thromboembolic complications occurred in $5(7.3 \%)$ of the treated aneurysms and $3(4.3 \%)$ were symptomatic. Access-site complications occurred in $1(1.4 \%)$ aneurysm. Good clinical outcome at the last follow-up, defined as mRS Score 0 to 2, occurred in $97.6 \%$ of electively treated patients and $80 \%$ of patients with aneurysmal subarach- 
TABLE 1. Summary of clinical and demographic characteristics in 52 patients harboring 69 aneurysms

\begin{tabular}{|c|c|}
\hline Characteristic & Value $^{*}$ \\
\hline No. of aneurysms & 69 \\
\hline No. of patients & 52 \\
\hline Multiple aneurysms, no. of patients & 11 \\
\hline 2 aneurysms & $8(72.77)$ \\
\hline 3 aneurysms & $1(9.1)$ \\
\hline 4 aneurysms & $1(9.1)$ \\
\hline 5 aneurysms & $1(9.1)$ \\
\hline \multicolumn{2}{|l|}{ Sex, no. of patients } \\
\hline Male & $4(7.7)$ \\
\hline Female & $48(92.3)$ \\
\hline Mean age, yrs & $54.1 \pm 13.7$ \\
\hline Smoker, no. of patients & $17(32.7)$ \\
\hline Hypertension, no. of patients & $22(42.3)$ \\
\hline Family history of aneurysm, no. of patients $†$ & $6(15.4)$ \\
\hline Mean maximum dimension (mm) & $4.9 \pm 1.6$ \\
\hline \multicolumn{2}{|l|}{ Side, no. of aneurysms } \\
\hline Right & $31(44.9)$ \\
\hline Left & $38(55.1)$ \\
\hline $\mathrm{SAH}$, no. of aneurysms & $6(8.7)$ \\
\hline Prior treatment, no. of aneurysms & $6(8.7)$ \\
\hline Endovascular & $5(7.2)$ \\
\hline Surgical clipping & $0(0.0)$ \\
\hline Endovascular embolization \& clipping & $1(1.5)$ \\
\hline \multicolumn{2}{|l|}{ Morphology, no. of aneurysms } \\
\hline Saccular & $65(94.2)$ \\
\hline Fusiform/dissecting & $4(5.8)$ \\
\hline Intramural thrombus, no. of aneurysms & $3(4.3)$ \\
\hline Adjunctive coils, no. of aneurysms & $2(2.9)$ \\
\hline \multicolumn{2}{|l|}{ No. of PEDs } \\
\hline 1 & $66(95.7)$ \\
\hline 2 & $3(4.3)$ \\
\hline \multicolumn{2}{|l|}{ Antiplatelet therapy, no. of aneurysms } \\
\hline $325 \mathrm{mg}$ aspirin daily $+75 \mathrm{mg}$ clopidogrel daily & $52(75.4)$ \\
\hline $325 \mathrm{mg}$ aspirin daily + $150 \mathrm{mg}$ clopidogrel daily & $2(2.9)$ \\
\hline $325 \mathrm{mg}$ aspirin daily $+10 \mathrm{mg}$ prasugrel daily & $5(7.2)$ \\
\hline $325 \mathrm{mg}$ aspirin daily + $90 \mathrm{mg}$ ticagrelor twice daily & $10(14.5)$ \\
\hline \multicolumn{2}{|l|}{ Platelet function assay, no. of aneurysms } \\
\hline Clopidogrel responder & $54(78.3)$ \\
\hline Clopidogrel nonresponder & $15(21.7)$ \\
\hline \multicolumn{2}{|l|}{ Radiographic outcome, no. of aneurysms } \\
\hline 6 mos $\ddagger$ & $54(78.3)$ \\
\hline $100 \%$ occlusion & $42(77.8)$ \\
\hline$\geq 90 \%$ occlusion & $3(5.6)$ \\
\hline$<90 \%$ occlusion & $9(16.6)$ \\
\hline Last follow-up $\ddagger$ & $54(78.3)$ \\
\hline
\end{tabular}

CONTINUED IN NEXT COLUMN ॥
» CONTINUED FROM PREVIOUS COLUMN

TABLE 1. Summary of clinical and demographic characteristics in 52 patients harboring 69 aneurysms

\begin{tabular}{|c|c|}
\hline Characteristic & Value $^{*}$ \\
\hline \multicolumn{2}{|l|}{ Radiographic outcome, no. of aneurysms (continued) } \\
\hline Last follow-up (mos)§ & $11.5(5.3-16)$ \\
\hline $100 \%$ occlusion & $44(81.5)$ \\
\hline$\geq 90 \%$ occlusion & $3(5.6)$ \\
\hline$<90 \%$ occlusion & $7(12.9)$ \\
\hline \multicolumn{2}{|l|}{ Complications of treated aneurysms } \\
\hline Thromboembolic complication & $5(7.3)$ \\
\hline Symptomatic thromboembolic complications & $3(4.3)$ \\
\hline Access-site complication & $1(1.4)$ \\
\hline \multicolumn{2}{|l|}{ Clinical outcome at last follow-up, no. of patients } \\
\hline \multicolumn{2}{|l|}{ Elective } \\
\hline Good outcome (mRS Score 0-2) & $40(97.6)$ \\
\hline Score 0 & $30(73.2)$ \\
\hline Score 1 & $7(17.1)$ \\
\hline Score 2 & $3(7.3)$ \\
\hline Poor outcome (mRS Score 3-6) & $1(2.4)$ \\
\hline Score 3 & $1(2.4)$ \\
\hline \multicolumn{2}{|l|}{$\mathrm{SAH}$} \\
\hline Good outcome (mRS Score 0-2) & $4(80)$ \\
\hline Score 0 & $1(20)$ \\
\hline Score 1 & $1(20)$ \\
\hline Score 2 & $2(40)$ \\
\hline Poor outcome (mRS Score 3-6) & $1(20)$ \\
\hline Score 3 & $1(20)$ \\
\hline
\end{tabular}

* Values are given as the number (\%) unless otherwise indicated. Mean values are presented as the mean \pm SD.

$\dagger$ Data were not available for 13 patients.

$\ddagger$ Data were not available for 15 aneurysms.

$\S$ Follow-up is reported as the median and interquartile range due to significant skewing in the data.

I Data were not available for 6 patients.

noid hemorrhage (SAH) (Table 1). One electively treated patient suffered a poor outcome (mRS Score 3). This patient experienced thrombosis of the left $\mathrm{A}_{1}$ segment of the anterior cerebral artery during their procedure. This was treated with eptifibatide with successful recanalization of the occluded segment. However, postprocedural imaging revealed SAH. At the last follow-up, the patient had no focal neurological deficits. The poor outcome (mRS Score 3 ) in 1 patient who presented with aneurysmal SAH was unrelated to the PED procedure. There were no cases of procedure-related mortality. The treatment of 2 aneurysms (3\%) resulted in transient visual deficits, and no patient experienced a permanent visual deficit (Table 2).

\section{Risk Factors Associated With the Anatomical Relationship Between OA Origin and Aneurysm}

The univariate analysis failed to associate any risk factor with a particular type of OA origin, with the exception of smoking $(\mathrm{p}=0.0162)$ which was most frequent in pa- 
TABLE 2. Anatomical relationship between OA origin and aneurysm, visual outcome, and OA patency

\begin{tabular}{lc}
\hline \multicolumn{1}{c}{ Characteristic } & No. of Aneurysms (\%) \\
\hline OA origin & \\
\hline Type 1 (OA separate from aneurysm) & $50(72.5)$ \\
\hline Type 2 (OA from aneurysm neck) & $12(17.4)$ \\
\hline Type 3 (OA from aneurysm dome) & $7(10.1)$ \\
\hline Visual outcome* & $2(3.0)$ \\
\hline Transient deficits & $0(0.0)$ \\
\hline Permanent deficits & $63(96.8)$ \\
\hline Patency of OA at last follow-up $\dagger$ & \\
\hline * Data were not available for 3 aneurysms. \\
$\dagger$ Data were not available for 6 aneurysms.
\end{tabular}

tients with Type 1 aneurysms. Specifically, aneurysm size $(\mathrm{p}=0.415)$, history of prior treatment $(\mathrm{p}=1)$, morphology $(\mathrm{p}=0.462)$, and number of PEDs $(\mathrm{p}=0.626)$ were not associated with the type of OA origin. There was an even distribution of antiplatelet regimens $(p=0.499)$ and rate of clopidogrel nonresponders $(\mathrm{p}=0.160)$ between the 3 types (Table 3).

\section{Outcome and Anatomical Relationship of OA Origin and Aneurysm}

Type $3 \mathrm{OA}$ origin was associated with a lower rate of complete aneurysm occlusion $(p=0.0297)$. There was also a trend toward visual deficits $(\mathrm{p}=0.0797)$ and a lower rate of OA patency $(\mathrm{p}=0.0783)$ in Type 3 OA origin. Clinical outcome, as defined by mRS score, was not associated with any type of OA origin. The follow-up duration was significantly shorter in Type 3 OA origin $(p=0.00245)$ (Table 4).

\section{Discussion}

Visual outcome and OA patency are well-recognized measures for assessing the safety of PED treatment of paraophthalmic aneurysms. ${ }^{13,15,16,19}$ Despite this, little is known about the implications of the anatomical relationship between the OA origin and aneurysm. Particularly in small paraophthalmic aneurysms, the OA is known to originate from the aneurysm dome, rendering these aneurysms unsuitable for coil embolization. Utilizing a multicenter cohort, we identified 69 small paraophthalmic aneurysms and assessed the radiographic and clinical outcomes with an emphasis on the anatomical relationship between the OA origin and aneurysm. In $10.1 \%$ of aneurysms, the OA originated from the aneurysm dome (Type 3 ), in $17.4 \%$ the OA originated from the neck (Type 2), and in $72.5 \%$ the OA originated from the ICA separate from the aneurysm (Type 1). We did not identify the risk factors associated with a specific type of OA origin configuration, with the exception of smoking which was most frequent in Type 1 . Complete to near-complete occlusion was achieved in $87.1 \%$ of treated aneurysms, and the OA remained patent in $96.8 \%$. Good clinical outcome was observed in $97.6 \%$ of electively treated patients and $80 \%$ of patients who presented with aneurysmal SAH. No permanent and $2(3 \%)$ transient visual deficits occurred; these patients underwent a formal neuro-ophthalmological evaluation and did not require intervention. Type $3 \mathrm{OA}$ origin was associated with a lower rate of complete aneurysm occlusion, a trend toward visual deficits, and a lower rate of OA patency. The follow-up of aneurysms with Type 3 OA origin was short, and long-term follow-up may potentially show an increased rate of aneurysm occlusion. The second antiplatelet agent was discontinued at the 6-month follow-up to facilitate aneurysm thrombosis. Clinical outcome, as measured by mRS, was not associated with any type of OA origin.

\section{Outcome in Paraophthalmic Aneurysms Treated With PED}

The rate of OA patency is in line with other series. Chalouhi et al. reported an $89 \%$ OA patency rate in 95 patients treated with PED for paraophthalmic aneurysm. ${ }^{1}$ Other series have reported that the OA remained patent in $79 \%$ to $96 \%$ of patients. ${ }^{13,15,21}$ The correlation of OA patency with visual outcome after PED placement is challenging due to the wide variability in collateral blood supply and inconsistent postprocedural ophthalmological evaluations. Moreover, in cases in which proximal flow in the OA is reduced after PED, retrograde filling of the OA may occur via external carotid artery collaterals. In fact, the presence of relatively good OA collaterals raises the possibility that retinal ischemia following the endovascular treatment of paraophthalmic aneurysms is more likely due to a thromboembolic mechanism, rather than reduction in antegrade OA flow. We were unable, however, to identify any correlation between visual deficits and clopidogrel responder status $(\mathrm{p}=1)$. Outside of research studies, patients are not routinely committed to formal neuroophthalmological testing in routine clinical practice unless compromised visual function is either reported by the patient or noted on gross visual examination. ${ }^{1}$ The present study used a similar paradigm and identified transient and permanent visual deficits in $3 \%$ and $0 \%$ of patients, respectively. This is comparable to other studies that used a similar protocol and reported visual deficits in $0 \%^{13,15}$ to $1 \%^{1}$ of patients and similar or superior to visual outcomes after treating aneurysms with traditional endovascular techniques ${ }^{3,4,8}$ or microsurgical clipping. ${ }^{8,14}$ Approximately two-thirds of patients with preexisting visual deficits from large and giant cavernous and supraclinoid ICA aneurysms who were enrolled in the Pipeline for Uncoilable or Failed Aneurysms trial had neuroophthalmological examinations that showed improvement in visual function. A preexisting deficit worsened in 1 patient $(2.6 \%)$, and $5 \%$ of patients developed a new deficit. ${ }^{17}$ The findings in the aforementioned studies stand in strong contrast to 1 study that found coverage of the OA with PED resulted in transient and permanent visual complications in $21.4 \%$ and $17.9 \%$ of patients, respectively. All patients underwent a complete and extensive neuroophthalmological examination, including retinal photography, retinal fluorescein angiography in selected cases, and optical coherence tomography of the central foveal area. ${ }^{16}$ This study is also the only study that investigated the association of OA origin and outcomes in 
TABLE 3. Risk factors associated with the anatomical relationship between OA origin and aneurysm*

\begin{tabular}{|c|c|c|c|c|}
\hline Characteristic & $\begin{array}{c}\text { Type } 1(\mathrm{OA} \text { Separate } \\
\text { From Aneurysm) }(\mathrm{n}=50)\end{array}$ & $\begin{array}{c}\text { Type } 2 \text { (OA From } \\
\text { Aneurysm Neck) }(n=12)\end{array}$ & $\begin{array}{c}\text { Type } 3 \text { (OA from } \\
\text { Aneurysm Dome) }(n=7)\end{array}$ & p Value \\
\hline \multicolumn{5}{|l|}{ Sex } \\
\hline Male & $3(6.0)$ & $2(16.7)$ & $1(14.3)$ & 0.244 \\
\hline Female & $47(94.0)$ & $10(83.3)$ & $6(85.7)$ & \\
\hline Mean age \pm standard deviation (yrs) & $53.3 \pm 13.5$ & $53.3 \pm 14.3$ & $51.0 \pm 17.3$ & 0.922 \\
\hline Smoker & $25(50.0)$ & $3(25.0)$ & $0(0)$ & 0.0162 \\
\hline Hypertension & $25(50.0)$ & $6(50.0)$ & $1(14.3)$ & 0.222 \\
\hline Family history of aneurysm $\dagger$ & $9(23.7)$ & $3(30.0)$ & $0(0)$ & 0.641 \\
\hline Size $(\mathrm{mm})$ & $4.6 \pm 1.8$ & $5.3 \pm 1.6$ & $4.9 \pm 1.0$ & 0.415 \\
\hline \multicolumn{5}{|l|}{ Side } \\
\hline Right & $21(42.0)$ & $6(50.0)$ & $4(57.1)$ & 0.791 \\
\hline Left & $29(58.0)$ & $6(50.0)$ & $3(42.9)$ & \\
\hline $\mathrm{SAH}$ & $6(12.0)$ & $0(0.0)$ & $0(0 \%)$ & 0.627 \\
\hline \multicolumn{5}{|l|}{ Prior treatment } \\
\hline None & $45(90.0)$ & $11(91.7)$ & $7(100.0)$ & 1 \\
\hline Endovascular embolization & $4(8.0)$ & $1(8.3)$ & $0(0.0)$ & \\
\hline Surgical clipping & $0(0.0)$ & $0(0.0)$ & $0(0.0)$ & \\
\hline Endovascular embolization \& clipping & $1(2.0)$ & $0(0.0)$ & $0(0.0)$ & \\
\hline \multicolumn{5}{|l|}{ Morphology } \\
\hline Saccular & $47(94.0)$ & $12(100.0)$ & $6(85.7)$ & 0.462 \\
\hline Fusiform/dissecting & $3(6.0)$ & $0(0.0)$ & $1(14.3)$ & \\
\hline Intramural thrombus & $3(6.0)$ & $0(0)$ & $0(0)$ & 1 \\
\hline Adjunctive coils & $1(2.0)$ & $1(8.3)$ & $0(0)$ & 0.478 \\
\hline \multicolumn{5}{|l|}{ No. of PEDS } \\
\hline 1 & $48(96.0)$ & $11(91.7)$ & $7(100.0)$ & 0.626 \\
\hline 2 & $2(4.0)$ & $1(8.3)$ & $0(0.0)$ & \\
\hline \multicolumn{5}{|l|}{ Antiplatelet therapy } \\
\hline $325 \mathrm{mg}$ aspirin daily $+75 \mathrm{mg}$ clopidogrel daily & $38(76.0)$ & $10(83.3)$ & $4(57.1)$ & 0.499 \\
\hline $325 \mathrm{mg}$ aspirin daily + $150 \mathrm{mg}$ clopidogrel daily & $2(4.0)$ & $0(0.0)$ & $0(0.0)$ & \\
\hline $325 \mathrm{mg}$ aspirin daily $+10 \mathrm{mg}$ prasugrel daily & $4(8.0)$ & $1(8.3)$ & $0(0.0)$ & \\
\hline $325 \mathrm{mg}$ aspirin daily + $90 \mathrm{mg}$ ticagrelor twice daily & $6(12.0)$ & $1(8.3)$ & $3(42.9)$ & \\
\hline \multicolumn{5}{|l|}{ Platelet function assay } \\
\hline Clopidogrel responder & $36(72.0)$ & $11(91.7)$ & $7(100.0)$ & 0.16 \\
\hline Clopidogrel nonresponder & $14(28.0)$ & $1(8.3)$ & $0(0.0)$ & \\
\hline
\end{tabular}

* Values are given as the number of aneurysms (\%) unless otherwise indicated. Mean values are presented as the mean \pm SD.

$\dagger$ Data were not available for 17 aneurysms.

28 patients with small aneurysms (median maximum aneurysm diameter $8 \mathrm{~mm}$ ). The authors did not identify an association between the location of the OA origin and aneurysm occlusion rates, which is potentially a result of the small sample size. Retinal emboli and visual deficits occurred most commonly in patients with Type 3 OA origin, which is consistent with the present series. Those authors postulate that for Type 3 OA origin, restoration of the laminar flow with coil embolization prior to PED placement may be warranted..$^{16}$ Due to the small size of most of these Type 3 aneurysms and the inherent risk of acute OA occlusion with coil placement, we question whether this approach is viable and would not recommend it. With the exception of Type 3 OA configurations, coil embolization with or without balloon or stent assistance of paraophthalmic artery aneurysms carries a low risk of visual impairment. ${ }^{3}$ Good clinical outcomes were reported in $95 \%$ of patients with these traditional endovascular techniques ${ }^{3}$ and are comparable to the experience in the present series. However, the lower rate of recurrence and need for retreatment ${ }^{3}$ would favor PED as the treatment of choice when available.

\section{Rationale for the Treatment of Small Aneurysms With PED}

Elective treatment of small aneurysms that are less than $7 \mathrm{~mm}$ across the largest dimension remains a controversial issue. Natural history data from the International Study of Unruptured Intracranial Aneurysms reveal 5-year cumu- 
TABLE 4. Outcomes and anatomical relationship between ophthalmic artery origin and aneurysm*

\begin{tabular}{|c|c|c|c|c|}
\hline Variable & $\begin{array}{c}\text { Type } 1(\text { OA Separate } \\
\text { From Aneurysm) }(n=50)\end{array}$ & $\begin{array}{c}\text { Type } 2 \text { (OA From } \\
\text { Aneurysm Neck) }(n=12)\end{array}$ & $\begin{array}{c}\text { Type } 3 \text { (OA from } \\
\text { Aneurysm Dome) }(n=7)\end{array}$ & $\mathrm{p}$ Value \\
\hline \multicolumn{5}{|l|}{ Visual outcome $\dagger$} \\
\hline Deficit & $0(0.0)$ & $1(8.3)$ & $1(14.3)$ & 0.0797 \\
\hline No deficit & $47(100)$ & $11(91.7)$ & $6(85.7)$ & \\
\hline Ophthalmic artery patency $\ddagger$ & $45(100)$ & $10(90.9)$ & $6(85.7)$ & 0.0783 \\
\hline \multicolumn{5}{|l|}{ Occlusion at the last follow-up§ } \\
\hline Complete $(100 \%)$ & $34(89.5)$ & $7(70.0)$ & $3(50.0)$ & 0.0297 \\
\hline Incomplete $(<100 \%)$ & $4(10.5)$ & $3(30.0)$ & $3(50.0)$ & \\
\hline Mean follow-up duration \pm standard deviation (mos.)ף & $10.1 \pm 8.0$ & $20.5 \pm 13.5$ & $6.5 \pm 4.3$ & 0.00245 \\
\hline \multicolumn{5}{|l|}{$m R S^{* *}$} \\
\hline $0-2$ & $42(93.3)$ & $12(100)$ & $7(100)$ & 0.843 \\
\hline $3-6$ & $3(6.7)$ & $0(0.0)$ & $0(0.0)$ & \\
\hline $\begin{array}{l}\text { * Values are given as the mean } \pm \text { SD unless otherwise indicat } \\
\dagger \text { Data were not available for } 3 \text { aneurysms. } \\
\text { f Data were not available for } 6 \text { aneurysms. } \\
\S \text { Data were not available for } 15 \text { aneurysms. } \\
\text { I Data were not available for } 14 \text { aneurysms. } \\
* * \text { Data were not available for } 5 \text { aneurysms. }\end{array}$ & & & & \\
\hline
\end{tabular}

lative rupture rates of $1.5 \%$ and $0 \%$ for anterior circulation aneurysms in patients with and without a history of SAH, respectively. ${ }^{20}$ The Unruptured Cerebral Aneurysm Study (UCAS) found an annual rupture rate of $0.14 \%$ for paraclinoid ICA aneurysms measuring 3 to $4 \mathrm{~mm}$ in a Japanese cohort. ${ }^{18}$ Both studies, however, may have been influenced by treatment selection bias. In UCAS, $69.7 \%$ of all aneurysms selected for treatment were less than 7 $\mathrm{mm}$ in maximum diameter. Of the ICA aneurysms, including paraophthalmic aneurysms, 44\% were treated and only $56 \%$ were observed. ${ }^{18}$ Furthermore, the average size of the ruptured aneurysms is between 6 and $7 \mathrm{~mm} .^{7,12} \mathrm{~Pa}-$ tients with risk factors including female sex, young age, smoking, hypertension, history of $\mathrm{SAH}$, and high risk aneurysm morphology (e.g., daughter sac, size progression) are commonly selected for treatment. ${ }^{2,9}$ Patients undergoing treatment for a small aneurysm in the current series represent only a fraction of the treated aneurysms at the participating centers; the majority of small aneurysms are followed expectantly. To overcome this issue, we collected data from 4 major academic institutions in order to arrive at a representative number of paraophthalmic aneurysms that were treated with PED.

\section{Limitations}

Short follow-up is an important limitation of the present study. While aneurysms with Type 1 OA origin were associated with a complete occlusion rate of almost $89.5 \%$ at 10.1 months post-PED placement, only $50 \%$ of aneurysms with Type 3 OA were completely occluded at 6.5 months. Longer follow-up may potentially show an increased rate of complete occlusion in these aneurysms, particularly with the discontinuation of the second antiplatelet agent. Other limitations include data collection and imaging interpretation at the individual centers, as opposed to a central location.

\section{Conclusions}

PED treatment of small paraophthalmic aneurysms is safe and effective. Aneurysm where the OA arises from the aneurysm dome may be associated with lower rates of aneurysm occlusion and OA patency and higher rates of transient visual complications.

\section{References}

1. Chalouhi N, Daou B, Kung D, Zanaty M, Phillips JL, Tjoumakaris S, et al: Fate of the ophthalmic artery after treatment with the Pipeline Embolization Device. Neurosurgery 77:581-584, 2015

2. Chalouhi N, Zanaty M, Whiting A, Yang S, Tjoumakaris S, Hasan D, et al: Safety and efficacy of the Pipeline Embolization Device in 100 small intracranial aneurysms. J Neurosurg 122:1498-1502, 2015

3. D’Urso PI, Karadeli HH, Kallmes DF, Cloft HJ, Lanzino G: Coiling for paraclinoid aneurysms: time to make way for flow diverters? AJNR Am J Neuroradiol 33:1470-1474, 2012

4. Ferrell AS, Lessne ML, Alexander MJ, Shah P, Golshani K, Zomorodi A, et al: Visual complications after stent-assisted endovascular embolization of paraophthalmic and suprasellar variant superior hypophyseal aneurysms: the Duke Cerebrovascular Center experience in 57 patients. World Neurosurg 78:289-294, 2012

5. Fiorella D, Lylyk P, Szikora I, Kelly ME, Albuquerque FC, McDougall CG, et al: Curative cerebrovascular reconstruction with the Pipeline embolization device: the emergence of definitive endovascular therapy for intracranial aneurysms. J Neurointerv Surg 1:56-65, 2009

6. Gross BA, Du R: Natural history of cerebral arteriovenous malformations: a meta-analysis. J Neurosurg 118:437-443, 2013

7. Harrigan M, Deveikis J: Intracranial aneurysms and subarachnoid hemorrhage, in Handbook of Cerebrovascular Disease and Neurointerventional Technique. New York: Humana Press, Springer, 2009, p 443

8. Hoh BL, Carter BS, Budzik RF, Putman CM, Ogilvy CS: Re- 
sults after surgical and endovascular treatment of paraclinoid aneurysms by a combined neurovascular team. Neurosurgery 48:78-90, 2001

9. Korja M, Lehto H, Juvela S: Lifelong rupture risk of intracranial aneurysms depends on risk factors: a prospective Finnish cohort study. Stroke 45:1958-1963, 2014

10. Kulcsár Z, Ernemann U, Wetzel SG, Bock A, Goericke S, Panagiotopoulos V, et al: High-profile flow diverter (silk) implantation in the basilar artery: efficacy in the treatment of aneurysms and the role of the perforators. Stroke 41:1690 1696, 2010

11. Lawton MT, Gress D, Higashida RT: Controversies in Neurological Surgery: Neurovascular Diseases. New York: Thieme, 2011

12. McDougall CG, Spetzler RF, Zabramski JM, Partovi S, Hills NK, Nakaji P, et al: The Barrow Ruptured Aneurysm Trial. J Neurosurg 116:135-144, 2012

13. Moon K, Albuquerque FC, Ducruet AF, Webster Crowley R, McDougall CG: Treatment of ophthalmic segment carotid aneurysms using the pipeline embolization device: clinical and angiographic follow-up. Neurol Res 36:344-350, 2014

14. Nanda A, Javalkar V: Microneurosurgical management of ophthalmic segment of the internal carotid artery aneurysms: single-surgeon operative experience from Louisiana State University, Shreveport. Neurosurgery 68:355-371, 2011

15. Puffer RC, Kallmes DF, Cloft HJ, Lanzino G: Patency of the ophthalmic artery after flow diversion treatment of paraclinoid aneurysms. J Neurosurg 116:892-896, 2012

16. Rouchaud A, Leclerc O, Benayoun Y, Saleme S, Camilleri Y, D'Argento F, et al: Visual outcomes with flow-diverter stents covering the ophthalmic artery for treatment of internal carotid artery aneurysms. AJNR Am J Neuroradiol 36:330336,2015

17. Sahlein DH, Fouladvand M, Becske T, Saatci I, McDougall CG, Szikora I, et al: Neuroophthalmological outcomes associated with use of the Pipeline Embolization Device: analysis of the PUFS trial results. J Neurosurg 123:897-905, 2015

18. Morita A, Kirino T, Hashi K, Aoki N, Fukuhara S, Hashimoto $\mathrm{N}$, et al: The natural course of unruptured cerebral aneurysms in a Japanese cohort. N Engl J Med 366:2474-2482, 2012
19. Vedantam A, Rao VY, Shaltoni HM, Mawad ME: Incidence and clinical implications of carotid branch occlusion following treatment of internal carotid artery aneurysms with the pipeline embolization device. Neurosurgery 76:173-178, 2015

20. Wiebers DO, Whisnant JP, Huston J III, Meissner I, Brown RD Jr, Piepgras DG, et al: Unruptured intracranial aneurysms: natural history, clinical outcome, and risks of surgical and endovascular treatment. Lancet 362:103-110, 2003

21. Zanaty M, Chalouhi N, Barros G, Schwartz EW, Saigh MP, Starke RM, et al: Flow-diversion for ophthalmic segment aneurysms. Neurosurgery 76:286-290, 2015

\section{Disclosures}

Dr. Mocco is a consultant for Lazurus Effect, Reverse Medical, Pulsar, Edge Therapeutics, and Medina and has a financial relationship with Blockade Medical. Dr. Thomas has a financial relationship with Stryker and a nonfinancial relationship with the Data Safety Monitoring Board of the SCENT flow diverter trial.

\section{Author Contributions}

Conception and design: Griessenauer, Ogilvy, Thomas. Acquisition of data: Griessenauer, Foreman, Stapleton, He, Thomas. Analysis and interpretation of data: Griessenauer, Ogilvy, Foreman, Chua, Harrigan, Stapleton, Patel, He, Thomas. Drafting the article: Griessenauer. Critically revising the article: all authors. Reviewed submitted version of manuscript: all authors. Approved the final version of the manuscript on behalf of all authors: Griessenauer. Statistical analysis: Griessenauer, Chua. Administrative/ technical/material support: Griessenauer, Ogilvy, Thomas. Study supervision: Griessenauer, Ogilvy, Thomas.

\section{Correspondence}

Christoph J. Griessenauer, Neurosurgical Service, Beth Israel Deaconess Medical Center, Harvard Medical School, 110 Francis St., Ste.3B, Boston, MA 02215. email: christoph.griessenauer@ gmail.com. 